\title{
Ontogenetic shifts in the trophic ecology of two alvinocaridid shrimp species at hydrothermal vents on the Mariana Arc, western Pacific Ocean
}

\author{
Catherine J. Stevens ${ }^{1,4, *}$, Helene Limén ${ }^{1,5}$, David W. Pond ${ }^{2}$, Yves Gélinas ${ }^{3}$, \\ S. Kim Juniper ${ }^{1,6}$ \\ ${ }^{1}$ Centre GÉOTOP-UQÀM-McGill, Université du Québec à Montréal, CP 8888 Succursale Centre-Ville, Montréal, \\ Québec H3C 3P8, Canada \\ ${ }^{2}$ Biological Sciences Division, British Antarctic Survey, NERC, High Cross, Madingley Road, Cambridge CB3 0ET, UK \\ ${ }^{3}$ Chemistry and Biochemistry Department, Concordia University, Loyola Science Complex, 7141 Sherbrooke St. W., \\ Montréal, Québec H4B 1R6, Canada \\ ${ }^{4}$ Present address: National Institute of Water and Atmospheric Research, Private Bag 14-901, 301 Evans Bay Parade, \\ Greta Point, Wellington, New Zealand \\ ${ }^{5}$ Present address: Research Service, The Riksdag Administration, The Swedish Parliament, 10012 Stockholm, Sweden \\ ${ }^{6}$ Present address: School of Earth \& Ocean Sciences and Department of Biology, University of Victoria, PO Box 3055 \\ Victoria, British Columbia V8W 3N5, Canada
}

\begin{abstract}
The Mariana Arc of submarine volcanoes has recently been the site of an international, interdisciplinary study into the structure and function of the associated hydrothermal systems. A broad size range of juvenile alvinocaridid shrimp, Opaepele loihi and Alvinocaris sp. M (diagnosis in preparation by R. Webber), were collected from active sites on NW Rota-1 and NW Eifuku volcanoes. Fatty acid biomarkers and stable isotopes of carbon and nitrogen revealed a photosynthetic signal in small juveniles of both species, probably acquired during a pelagic larval phase. Size-related changes in the fatty acid composition of both species indicated a dietary switch from pelagic feeding on photosynthetic material to ingestion of bacteria at vent sites after settlement. This is especially true for $O$. loihi, where carbon isotopic signatures implied ingestion of bacteria with form II RuBisCo. Juvenile Alvinocaris sp. M also appear to have eaten bacteria, although probably those with form I RuBisCo; detritus may also feature in their diet at an early stage. With increasing size, the fatty acid and isotopic composition of Alvinocaris sp. M implied a lesser dependence on bacterivory and a possible switch to carnivory. Generally, Alvinocaris sp. $\mathrm{M}$ and $\mathrm{O}$. loihi are more similar in their biochemical composition to opportunistic alvinocaridids than to strict bacterivores. We suggest that as juveniles both species rely to varying degrees on bacteria and that opportunism and scavenging are likely sources of nutrition in older individuals.
\end{abstract}

KEY WORDS: Hydrothermal vent $\cdot$ Shrimp $\cdot$ Alvinocaridid $\cdot$ Fatty acid $\cdot$ Stable isotope $\cdot$ Mariana Arc Western Pacific Ocean

Resale or republication not permitted without written consent of the publisher

\section{INTRODUCTION}

Alvinocaridid shrimp (Infraorder Caridea, Family Alvinocarididae), which include the genera Alvinocaris, Chorocaris, Mirocaris, Nautilocaris, Opaepele, Rimicaris, and Shinkaicaris, are endemic to hydrothermal vents and cold seeps (Martin \& Haney, 2005). Including the many undescribed species that have been reported, there are currently $\sim 27$ species of alvinocaridids at hydrothermal vents, over half of which belong to the genus Alvinocaris (Martin \& Haney 2005). While many aspects of the alvinocaridid life cycle are uncertain, it is reasonably clear that sedentary adults, found only at the vents themselves, release eggs or migratory larvae into the water column; juveniles later return to vents and settle, grow to sexual maturity, and reproduce (Allen et al. 1998, Dixon et al. 1998). 
Much of our knowledge of adult alvinocaridid trophic ecology stems from analysis of shrimp from the MidAtlantic Ridge (MAR), where the feeding habits of Rimicaris exoculata, Alvinocaris markensis, Mirocaris fortunata and Chorocaris chacei have been described (Van Dover et al. 1988, Casanova et al. 1993, Segonzac et al. 1993, Pond et al. 1997a,b, 2000, Allen et al. 1998, 2001, Polz et al. 1998, Gebruk et al. 2000, Vereshchaka et al. 2000). At MAR vent sites, alvinocaridid shrimp exist along a trophic continuum with respect to their feeding strategies. At one end is primary grazing of bacterial episymbionts ( $R$. exoculata) and at the other is opportunism and scavenging (A. markensis, $M$. fortunata) (Gebruk et al. 2000). C. chacei occupies an intermediate position since it gains nutrition from its episymbionts and can also feed as a scavenger (Segonzac et al. 1993, Gebruk et al. 2000).

Considerably less is known about the trophic ecology of alvinocaridids at vent sites in the Pacific and Indian Oceans, as large areas remain unexplored and few studies have been undertaken. Most of the published work involves determination of stable isotope compositions in shrimp with episymbionts: Chorocaris vandoverae (Van Dover \& Fry 1989) and Rimicaris kairei (Van Dover 2002) are primary consumers of episymbiotic bacteria on the Mariana Back-Arc Basin (western Pacific Ocean) and at the Rodriguez Triple Junction (Indian Ocean), respectively. Thus far, only one study has looked at the biogeography and ecology of non-symbiotic alvinocaridids in the Pacific Ocean, on the Mariana Arc (Tunnicliffe et al. unpubl. data).

The Mariana Arc consists of a series of shallow submarine volcanoes along the western edge of the Pacific Plate $\left(\sim 12^{\circ} \mathrm{N}\right.$ to $24^{\circ} \mathrm{N}, \sim 142^{\circ} \mathrm{E}$ to $\left.146^{\circ} \mathrm{E}\right)$. Opaepele loihi and Alvinocaris sp. M (diagnosis in preparation by R. Webber) were collected on NW Rota-1 and NW Eifuku volcanoes during extensive biological sampling in 2004. Throughout this period, both NW Rota-1 and NW Eifuku seamounts were highly active hydrothermally and NW Rota-1 also showed intensive, sustained volcanism (Embley et al. 2006). Trophic studies at active sites on NW Rota-1 and NW Eifuku have shown that the severe habitat conditions give rise to speciespoor communities with simple food webs and dominance by mobile species, including alvinocaridid shrimp (Limén \& Juniper 2006, Limén et al. 2006, Tunnicliffe et al. unpubl. data). Since the 2004 sampling season, O. loihi and Alvinocaris sp. M have been found at 3 other seamounts on the Mariana Arc (Nikko, Forecast and Seamount X, Tunnicliffe et al. unpubl. data).

The analysis of naturally occurring biomarkers has facilitated the study of feeding ecology in harsh, remote hydrothermal vent environments. Fatty acid biomarkers have been useful in elucidating trophic connections among vent organisms (e.g. Fullarton et al.
1995, Kharlamenko et al. 1995, Pranal et al. 1996, Pond et al. 1998, 2002, Limén et al. in press) and in showing how specific dietary components change with life history in alvinocaridids (Pond et al. 1997a, 2000, Allen et al. 2001). Relative to organisms from ecosystems based on photosynthesis, vent animals are rich in fatty acids of bacterial origin, reflecting the chemosynthetic nature of these food webs. Bacterial fatty acids include odd-numbered and/or branched chains, 16- and 18carbon $\omega 7, \omega 8$ and $\omega 9$ monounsaturates, and $\omega 4$ dienes (Pranal et al. 1996, Pond et al. 1997b, 1998, Zhang et al. 2005). Bacterial feeding indices based on levels of these fatty acids in consumer tissues can then be used to determine relative degrees of bacterivory. To obtain accurate estimates of invertebrate diets, researchers should use multiple fatty acids simultaneously, focus on the composition of neutral lipid, and collect data during periods of active feeding and growth (Dalsgaard et al. 2003, Stübing \& Hagen 2003). Neutral lipids of consumers generally reflect the composition of assimilated food, while polar lipids, serving a more structural function, have specific fatty acid quotas and more rigid compositions (Lee et al. 1971). Stable isotopes have been successfully used with fatty acids in hydrothermal vent food web studies (Pond et al. 1997a, 2000, Limén et al. in press). These 2 methods are complementary and together yield information that may be missed using either analysis alone (Nyssen et al. 2005). One trophic level has been equated to an enrichment of $+0.4 \%$ in ${ }^{13} \mathrm{C}$ and of $+3.4 \%$ in ${ }^{15} \mathrm{~N}$ between predator and prey tissues (Post 2002).

In this article, we present fatty acid and stable carbon and nitrogen isotopic compositions of Opaepele loihi and Alvinocaris sp. M from NW Eifuku, and O. loihi from NW Rota-1. As juveniles of many different sizes were collected, we explore dietary changes over the post-settlement period of these 2 species. Our data on ontogenetic changes in diet and feeding strategy shed light on how these species meet their energetic needs on the Mariana Arc and we compare these data to those of their congeners on the MAR.

\section{MATERIALS AND METHODS}

Field sampling. Samples were collected in March and April 2004 aboard the RV 'Thomas G. Thompson' as part of the Submarine Ring of Fire expedition to the Mariana Arc. Shrimp samples were taken with a suction sampler coupled to the remotely operated vehicle 'ROPOS' at submarine volcanoes NW Rota-1 $\left(14^{\circ} 36.05^{\prime} \mathrm{N}, 144^{\circ} 46.56^{\prime} \mathrm{E}\right)$ and NW Eifuku $\left(21^{\circ} 29.26^{\prime} \mathrm{N}, 144^{\circ} 02.49^{\prime} \mathrm{E}\right)$ (Fig. 1, Table 1). All sampling sites at NW Rota-1 (at Shimmering Vent and near Gastros) were on the central peak, which was charac- 


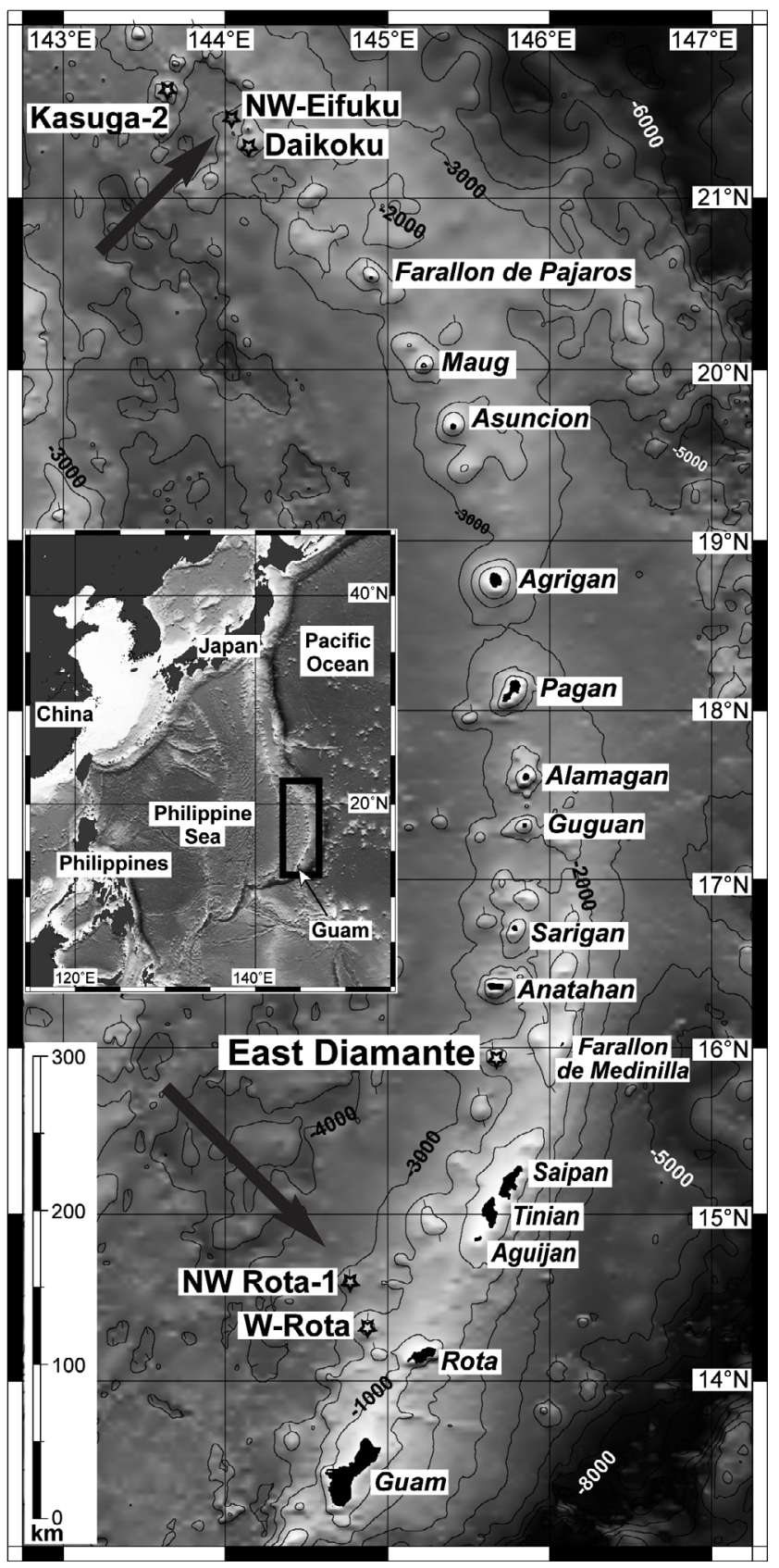

Fig. 1. Location of NW Eifuku and NW Rota-1 submarine volcanoes (large arrows) on the Mariana Arc. Image courtesy of S. Merle, NOAA Vents Program

terized by sustained volcanic activity and diffuse hydrothermal venting (Limén et al. 2006). The 2 sampling sites at NW Eifuku (at Champagne and near Fouling) were characterized by intense diffuse and focused hydrothermal flow (Limén \& Juniper 2006). The Champagne site was characterized by several white smokers and seafloor vents emitting fluids supersaturated with $\mathrm{CO}_{2}$ (Limén \& Juniper 2006). Descriptions of the biological communities at these sites are found elsewhere
(Limén \& Juniper 2006, Limén et al. 2006, Tunnicliffe et al. unpubl. data). Sorting of faunal samples took place on board and shrimp were provisionally identified to species and then frozen individually in aluminum foil. Northwest of the active peak on NW Rota1, particulate matter from an altitude of $30 \mathrm{~m}$ was concentrated onto a $142 \mathrm{~mm}$ GF/F filter by McLane pump (1250 1). Using a CTD-rosette, water was collected at $100 \mathrm{~m}$ and $13 \mathrm{l}$ was filtered onto a $47 \mathrm{~mm}$ $\mathrm{GF} / \mathrm{F}$; one half was analysed for isotopes, the other half for lipids. Detritus from NW Rota-1 was collected using the 'ROPOS' suction sampler and meiofauna were removed in the laboratory before biochemical analysis. All other end-member data (i.e. potential food sources of shrimp) reported in this study (see Fig. 4) are from Limén \& Juniper (2006) and Limén et al. (2006). Samples were stored at $-80^{\circ} \mathrm{C}$ before analysis.

Stable isotope analysis. In the laboratory, shrimp species identifications were verified using rostrum shape and structure, and total length (telson to tip of rostrum) measured for each (Table 1). Exoskeleton from the abdomen was removed and a sample of muscle was taken. All analyses were performed on tissue from individual shrimp and the unused material was refrozen at $-80^{\circ} \mathrm{C}$. Shrimp tissue samples and filter pieces with particulates were acidified in glass vials with $0.1 \mathrm{~N} \mathrm{HCl}$ to remove carbonates, rinsed once with Milli-Q water, and dried at $55^{\circ} \mathrm{C}$ for $24 \mathrm{~h}$. Stable carbon $\left({ }^{13} \mathrm{C} /{ }^{12} \mathrm{C}\right)$ and nitrogen $\left({ }^{15} \mathrm{~N} /{ }^{14} \mathrm{~N}\right)$ isotopic ratios were measured using a Micromass Isoprime isotope ratio mass spectrometer, in line with a Carlo Erba C/N element analyzer. Stable isotopic compositions are reported relative to Vienna Pee-Dee Belemnite and atmospheric nitrogen. Before isotopic analysis, $\mathrm{C}: \mathrm{N}$ ratios of detritus were determined to ascertain the mass of material needed; aliquots of detritus were acidified and rinsed directly in thick, smooth-walled tin capsules and carbon and nitrogen isotopes were measured separately. General linear models (simple linear regression) to describe relationships between shrimp isotopic composition and size were produced using Statistica 5.5A. To describe the fit of the regression model to our isotopic data, we used the 'adjusted' $\mathrm{r}^{2}$. 'Adjusted' ${ }^{2}$ values are more conservative than 'raw' ${ }^{2}$ ('adjusted' $\mathrm{r}^{2} \leq$ 'raw' $\mathrm{r}^{2}$ ), because they take into account the number of observations in the model.

Lipid analysis. Remaining shrimp tissues were placed in separate tubes containing $2 \mathrm{ml}$ chloroform, flushed with $\mathrm{N}_{2}$, sealed with Teflon tape and stored at $-20^{\circ} \mathrm{C}$ until analysis. The shrimp were ground with a metal rod and lipids were extracted according to Parrish (1999). Total lipid extracts were applied to silica gel columns $(\sim 0.8 \mathrm{~g}, 100-200$ mesh) that had been activated at $100^{\circ} \mathrm{C}$ for $1 \mathrm{~h}$. Following Budge \& Parrish (2003), neutral lipids were eluted with $8 \mathrm{ml}$ chloroform: 
Table 1. Opaepele loihi and Alvinocaris sp. M. Collections from the Mariana Arc submarine volcanoes

\begin{tabular}{|c|c|c|c|c|c|c|c|c|}
\hline Dive & $\begin{array}{l}\text { Date } \\
(2004)\end{array}$ & Site & Latitude & Longitude & $\begin{array}{l}\text { Depth } \\
\text { (m) }\end{array}$ & $\begin{array}{c}\text { Isotope and } \\
\text { lipid samples }\end{array}$ & Species & $\begin{array}{l}\text { Size } \\
(\mathrm{mm})\end{array}$ \\
\hline R782 & $28 \mathrm{March}$ & $\begin{array}{c}\text { NW Rota-1 } \\
\text { 'Shimmering Vent' }\end{array}$ & $14^{\circ} 36.07^{\prime} \mathrm{N}$ & $144^{\circ} 46.53^{\prime} \mathrm{E}$ & 515 & 20 & Opaepele loihi & $15.2-31.2$ \\
\hline R783 & 29 March & $\begin{array}{l}\text { NW Rota-1 } \\
\text { Near 'Gastros' }\end{array}$ & $14^{\circ} 36.05^{\prime} \mathrm{N}$ & $144^{\circ} 46.56^{\prime} \mathrm{E}$ & 527 & 13 & Opaepele loihi & $16.3-35.8$ \\
\hline R791 & 09 April & $\begin{array}{l}\text { NW Eifuku } \\
\text { 'Champagne' }\end{array}$ & $21^{\circ} 29.26^{\prime} \mathrm{N}$ & $144^{\circ} 24.84^{\prime} \mathrm{E}$ & 1608 & 4 & Opaepele loihi & $17.8-28.9$ \\
\hline R792 & 10 April & $\begin{array}{l}\text { NW Eifuku } \\
\text { Near 'Fouling' }\end{array}$ & $21^{\circ} 29.27^{\prime} \mathrm{N}$ & $144^{\circ} 25.08^{\prime} \mathrm{E}$ & 1594 & 11 & Alvinocaris sp. M & $17.0-36.0$ \\
\hline
\end{tabular}

NW ROTA-1 O O. loihi - Shimmering Vent
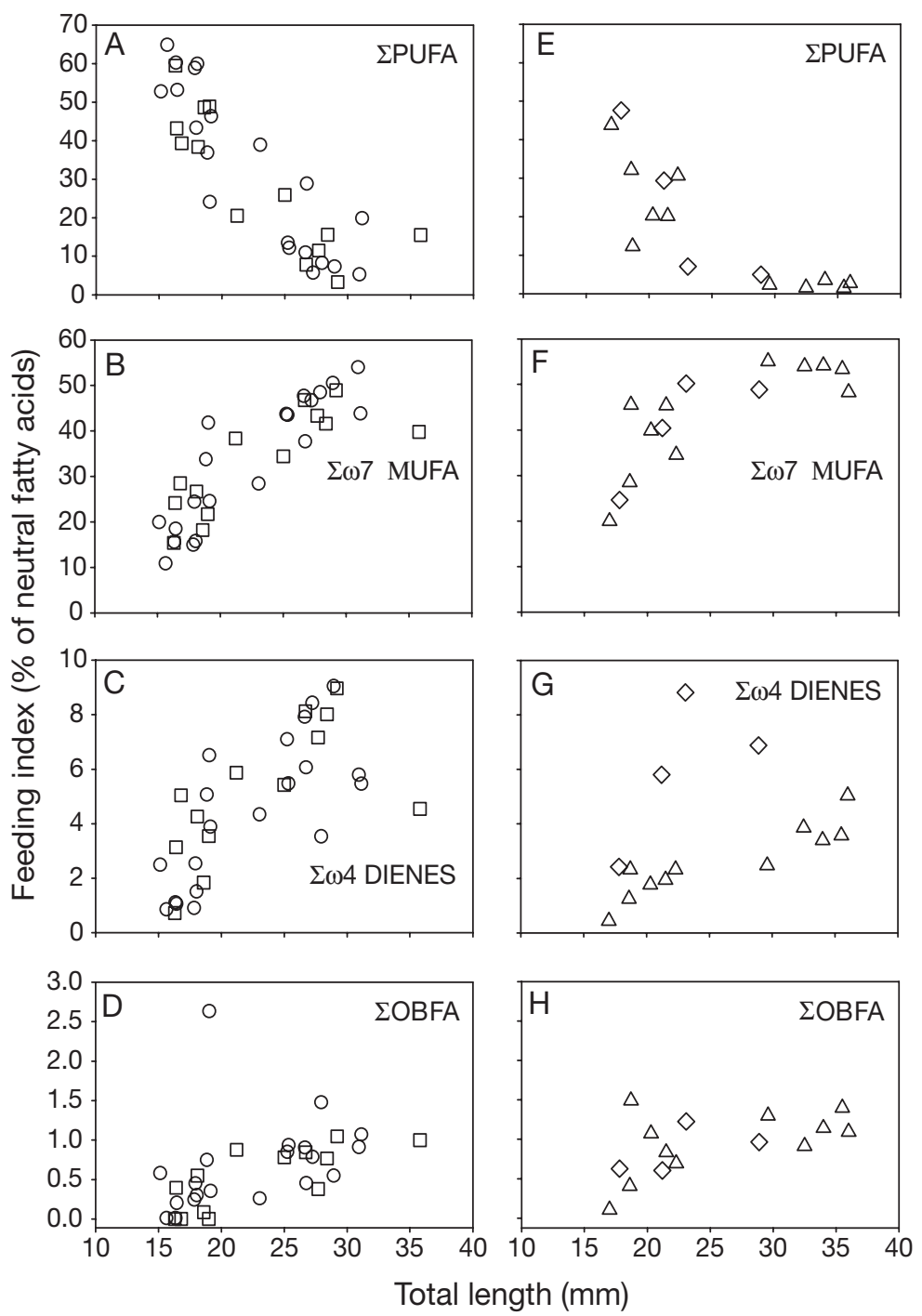

Fig. 2. Opaepele loihi and Alvinocaris sp. M. Relative PUFA content and lipid-based bacterial feeding indices (\% of neutral fatty acids) in juveniles at NW Rota-1 (A-D) and NW Eifuku (E-H) $(\Sigma \omega 7$ MUFA $=\Sigma 16: 1 \omega 7+$ $18: 1 \omega 7 ; \Sigma \omega 4$ dienes $=\Sigma 16: 2 \omega 4+18: 2 \omega 4)$ methanol:formic acid (98:1:0.5, v/v/v) and polar lipids were eluted with $6 \mathrm{ml}$ methanol. Lipid fractions were derivatized using $\mathrm{BF}_{3}$ methanol $\left(1.5 \mathrm{~h}, 85^{\circ} \mathrm{C}\right)$ and fatty acid methyl esters (FAME) were analyzed by flame-ionization detection on an Agilent Model 6890N gas chromatograph (GC) equipped with a DB5 column (30 m $\times 0.32 \mathrm{~mm} \times 0.25 \mu \mathrm{m})$. Helium was the carrier gas and the column was programmed as follows: $100^{\circ} \mathrm{C}$ (hold $1 \mathrm{~min}$ ), $214^{\circ} \mathrm{C}$ at $4^{\circ} \mathrm{C} \mathrm{min}^{-1}, 216^{\circ} \mathrm{C}$ at $0.5^{\circ} \mathrm{C} \mathrm{min}^{-1}$, $219^{\circ} \mathrm{C}$ at $4^{\circ} \mathrm{C} \mathrm{min}^{-1}, 223^{\circ} \mathrm{C}$ (hold $3 \mathrm{~min}$ ) at $0.5^{\circ} \mathrm{C} \mathrm{min}^{-1}, 270^{\circ} \mathrm{C}$ at $30^{\circ} \mathrm{C} \mathrm{min}^{-1}, 315^{\circ} \mathrm{C}$ (hold $10 \mathrm{~min}$ ) at $1.5^{\circ} \mathrm{C} \mathrm{min}^{-1}$. Many fatty acids could be identified using the commercial standards '37-Component,' 'PUFA No. 1' and 'Bacterial Acid Methyl Esters' (Supelco, SigmaAldrich). Further fatty acid characterization was performed using pentafluorobenzyl (PFB, Suhr et al. 2003) and picolinyl esters (Christie 2003). FAME were first separated into saturates, monounsaturates, dienes, and polyunsaturates by argentation high-performance thin-layer chromatography (Pond et al. 1998). The PFB and picolinyl esters were analyzed on a Thermo Finnigan GC/MS equipped with a wax column (Restek Stabilwax column, $30 \mathrm{~m} \times 0.25 \mathrm{~mm} \times 0.25 \mu \mathrm{m}$ ).

\section{RESULTS}

NW Rota-1: Lipids. Juvenile Opaepele loihi ranging in total length from 15.2 to $35.8 \mathrm{~mm}$ (Table 1) displayed marked ontogenetic changes in neutral lipid composition, with no noticeable differences between samples collected at Shimmering Vent and near Gastros (Fig. 2A; Tables 2 \& 3). The most obvious trend was a continual decrease in proportions of polyunsaturated fatty acids (PUFA) as total length increased. The thresh- 


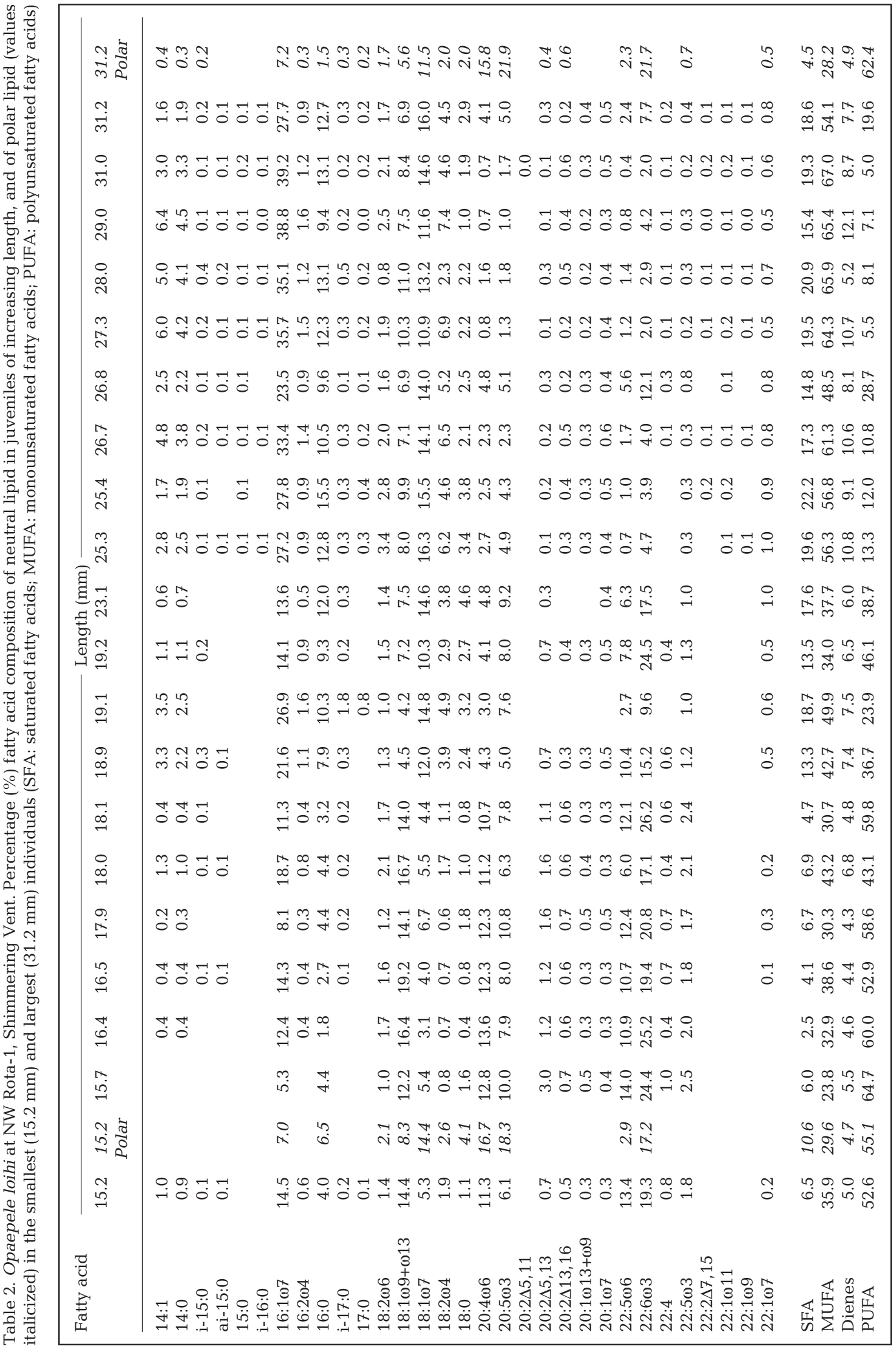


Table 3. Opaepele loihi juveniles at NW Rota-1, near Gastros. Percentage (\%) fatty acid composition of neutral lipid in juveniles of increasing length, and of polar lipid (values italicized) in the smallest $(16.3 \mathrm{~mm}$ ) and largest (35.8 mm) individuals (SFA: saturated fatty acids; MUFA: monounsaturated fatty acids; PUFA: polyunsaturated fatty acids)

\begin{tabular}{|c|c|c|c|c|c|c|c|c|c|c|c|c|c|c|c|}
\hline \multirow[t]{2}{*}{ Fatty acid } & \multirow[b]{2}{*}{16.3} & \multirow[b]{2}{*}{$\begin{array}{l}16.3 \\
\text { Polar }\end{array}$} & \multirow[b]{2}{*}{16.4} & \multirow[b]{2}{*}{16.8} & \multirow[b]{2}{*}{18.1} & \multirow[b]{2}{*}{18.6} & \multirow{2}{*}{$\begin{array}{c}-\mathrm{L} \\
19.0\end{array}$} & \multicolumn{2}{|c|}{ ngth (mm) } & \multirow[b]{2}{*}{26.7} & \multirow[b]{2}{*}{27.7} & \multirow[b]{2}{*}{28.4} & \multirow[b]{2}{*}{29.2} & \multirow[b]{2}{*}{35.8} & \multirow[b]{2}{*}{$\begin{array}{c}35.8 \\
\text { Polar }\end{array}$} \\
\hline & & & & & & & & 21.2 & 25.0 & & & & & & \\
\hline $14: 1$ & 0.5 & & 1.8 & 3.1 & 2.7 & 0.5 & 1.9 & 3.8 & 3.9 & 6.2 & 4.5 & 4.2 & 5.6 & 1.3 & 0.2 \\
\hline 14:0 & 0.4 & & 1.3 & 2.0 & 2.0 & 0.5 & 1.3 & 2.4 & 2.5 & 3.6 & 2.7 & 2.9 & 3.5 & 2.3 & 0.3 \\
\hline i-15:0 & & & & & 0.1 & & & 0.1 & 0.1 & 0.2 & & 0.2 & 0.2 & 0.1 & \\
\hline ai-15:0 & & & & & 0.1 & & & 0.1 & 0.1 & 0.1 & & 0.1 & 0.1 & 0.0 & \\
\hline 15:0 & & & & & 0.1 & & & & & 0.1 & & 0.1 & 0.1 & 0.2 & \\
\hline i-16:0 & & & & & & & & & & 0.1 & & 0.1 & & & \\
\hline $16: 1 \omega 7$ & 9.2 & 4.6 & 14.0 & 17.6 & 17.4 & 11.1 & 12.6 & 26.2 & 22.6 & 34.3 & 27.6 & 27.9 & 36.3 & 25.2 & 7.7 \\
\hline $16: 2 \omega 4$ & & & 0.6 & 0.8 & 0.8 & 0.4 & 0.6 & 1.1 & 1.0 & 1.5 & 0.9 & 1.1 & 1.3 & 0.6 & 0.2 \\
\hline 16:0 & 5.1 & 6.6 & 7.6 & 8.0 & 8.0 & 5.6 & 6.1 & 10.7 & 10.1 & 11.1 & 13.1 & 11.3 & 12.1 & 14.1 & 5.5 \\
\hline $\mathrm{i}-17: 0$ & & & 0.2 & & 0.2 & & & 0.5 & 0.5 & 0.4 & 0.3 & 0.3 & 0.6 & 0.3 & 0.1 \\
\hline $17: 0$ & & & 0.1 & & 0.1 & 0.1 & & 0.1 & 0.1 & 0.1 & 0.1 & 0.1 & 0.1 & 0.4 & 0.3 \\
\hline $18: 2 \omega 6$ & 1.5 & 2.3 & 1.5 & 1.3 & 1.7 & 1.5 & 1.3 & 2.1 & 1.6 & 2.0 & 2.5 & 2.6 & 2.5 & 2.3 & 1.6 \\
\hline $18: 1 \omega 9+\omega 13$ & 13.9 & 10.5 & 10.7 & 6.8 & 10.0 & 18.3 & 8.6 & 10.1 & 9.1 & 9.5 & 7.5 & 6.5 & 9.2 & 10.1 & 8.3 \\
\hline $18: 1 \omega 7$ & 6.2 & 12.1 & 10.1 & 10.9 & 9.3 & 7.1 & 9.1 & 12.1 & 11.8 & 12.5 & 15.8 & 13.7 & 12.6 & 14.6 & 12.1 \\
\hline $18: 2 \omega 4$ & 0.7 & 0.9 & 2.6 & 4.2 & 3.4 & 1.4 & 2.9 & 4.8 & 4.5 & 6.7 & 6.2 & 6.9 & 7.6 & 3.9 & 2.2 \\
\hline 18:0 & 2.2 & 4.7 & 2.9 & 3.3 & 2.2 & 2.1 & 2.7 & 3.1 & 3.5 & 2.0 & 4.9 & 3.1 & 2.2 & 3.5 & 3.5 \\
\hline $20: 4 \omega 6$ & 10.3 & 16.0 & 9.7 & 8.0 & 9.3 & 8.7 & 12.6 & 5.0 & 6.1 & 2.0 & 3.5 & 2.5 & 0.7 & 1.8 & 7.6 \\
\hline $20: 5 \omega 3$ & 10.5 & 20.1 & 7.5 & 7.6 & 6.1 & 9.5 & 6.7 & 4.5 & 6.3 & 1.8 & 3.9 & 5.1 & 1.4 & 7.9 & 26.9 \\
\hline $20: 2 \Delta 5,11$ & & & & & & & & & 0.5 & 0.2 & 0.2 & 0.3 & & 0.1 & \\
\hline $20: 2 \Delta 5,13$ & 0.9 & & 0.9 & 0.7 & 1.2 & 0.7 & 1.8 & 0.4 & 0.1 & 0.3 & 0.4 & 0.6 & 0.6 & 0.8 & 1.1 \\
\hline $20: 2 \Delta 13,16$ & & & 0.7 & 0.5 & 0.6 & 0.7 & 0.6 & 0.4 & 0.3 & & & & 0.1 & & 0.3 \\
\hline $20: 1 \omega 13+\omega 9$ & & & 0.9 & 0.4 & 0.9 & 0.6 & 0.4 & 0.4 & 0.7 & 0.2 & 0.4 & 0.7 & 0.4 & 1.6 & 1.1 \\
\hline $20: 1 \omega 7$ & & & 0.4 & 0.4 & 0.5 & 0.4 & 0.4 & 0.4 & 0.3 & 0.4 & 0.4 & 0.6 & 0.4 & 1.0 & 0.5 \\
\hline $22: 5 \omega 6$ & 12.1 & 3.4 & 7.7 & 6.1 & 7.9 & 9.3 & 10.6 & 2.9 & 4.8 & 1.4 & 0.7 & 1.2 & 0.1 & 0.3 & 0.9 \\
\hline $22: 6 \omega 3$ & 23.9 & 18.9 & 16.2 & 16.1 & 12.9 & 19.0 & 16.6 & 7.4 & 7.8 & 2.2 & 3.2 & 6.2 & 0.9 & 5.0 & 17.4 \\
\hline $22: 4$ & 0.6 & & 0.5 & 0.4 & 0.8 & 0.6 & 0.6 & 0.2 & 0.3 & 0.1 & & 0.1 & & 0.1 & 0.2 \\
\hline $22: 5 \omega 3$ & 2.1 & & 1.5 & 1.1 & 1.4 & 1.6 & 1.8 & 0.6 & 0.6 & 0.2 & 0.2 & 0.5 & 0.1 & 0.4 & 0.8 \\
\hline $22: 2 \Delta 7,15$ & & & & & & & & & & & & 0.2 & 0.1 & 0.7 & 0.2 \\
\hline $22: 1 \omega 11$ & & & & & & & & & & 0.0 & & 0.1 & 0.1 & 0.2 & \\
\hline $22: 1 \omega 9$ & & & & & & & & & & 0.1 & & 0.1 & 0.1 & 0.1 & \\
\hline $22: 1 \omega 7$ & & & 0.4 & 0.6 & 0.3 & 0.4 & 0.7 & 0.7 & 0.8 & 0.7 & 1.0 & 0.9 & 0.9 & 0.9 & 1.0 \\
\hline SFA & 7.7 & 11.3 & 12.1 & 13.3 & 12.8 & 8.3 & 10.1 & 17.1 & 16.9 & 17.6 & 21.2 & 18.1 & 18.9 & 21.0 & 9.6 \\
\hline MUFA & 29.8 & 27.2 & 38.3 & 39.8 & 41.1 & 38.3 & 33.8 & 53.7 & 49.2 & 63.9 & 57.1 & 54.6 & 65.6 & 55.0 & 31.1 \\
\hline Dienes & 3.1 & 3.2 & 6.3 & 7.6 & 7.7 & 4.8 & 7.3 & 8.8 & 8.0 & 10.7 & 10.2 & 11.7 & 12.3 & 8.5 & 5.5 \\
\hline $\begin{array}{l}\text { PUFA } \\
\text { PUF }\end{array}$ & 59.5 & 58.3 & 43.2 & 39.3 & 38.4 & 48.6 & 48.9 & 20.5 & 25.9 & 7.8 & 11.5 & 15.6 & 3.3 & 15.5 & 53.8 \\
\hline
\end{tabular}

old size for high PUFA content in O. loihi was $25 \mathrm{~mm}$; above this length, relative PUFA levels dropped sharply (Tables $2 \& 3$, last row). In specimens $\leq 25 \mathrm{~mm}$, PUFA accounted for $\sim 21$ to $65 \%$ of the neutral fatty acids and the dominant PUFA was invariably 22:6 $\omega 3$, ranging from 7.4 to $26.2 \%$ across both sampling sites.

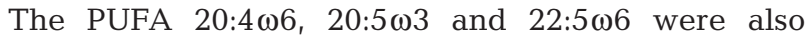
dominant in shrimp $\leq 25 \mathrm{~mm}$, while 22:4 and 22:5 $\mathrm{\omega 3}$ were less abundant, constituting $<3 \%$. The sizerelated decrease in proportions of PUFA in O. loihi corresponded to an increase in 3 bacterial feeding indices (Fig. 2B-D). Specifically, relative levels of $\omega 7$ monounsaturated fatty acids (MUFA) increased,

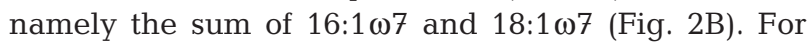
shrimp $>25 \mathrm{~mm}$, these 2 fatty acids together accounted for at least $30 \%$ of the neutral fatty acids. Also, as total length increased, so did the sum of the dienes 16:2 $\omega 4$

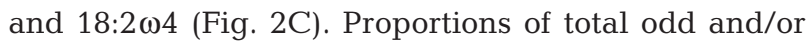
branched fatty acids (OBFA) also increased weakly with size (Fig. 2D). The polar lipid fraction of the 2 largest specimens (31.2 and $35.8 \mathrm{~mm}$ ) was rich in PUFA,

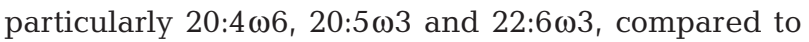
the PUFA-poor neutral lipid in these samples (Tables 2 $\& 3$, last 2 columns).

NW Rota-1: Stable isotopes. Juvenile Opaepele loihi had highly variable isotopic compositions, which were related to size (Fig. 3A,B). Individuals $>25 \mathrm{~mm}$, collected at both Shimmering Vent and near Gastros, were generally more enriched in ${ }^{13} \mathrm{C}$ and ${ }^{15} \mathrm{~N}$ than shrimp $\leq 25 \mathrm{~mm}$. O. loihi $\leq 25 \mathrm{~mm}$ exhibited a wide range of $\delta^{13} \mathrm{C}$ values ( -21.7 to $-10.1 \%$ ) and $\delta^{15} \mathrm{~N}$ values (6.6 to $10.6 \%$ ). There was a significant positive rela- 
NW ROTA-1


NW EIFUKU
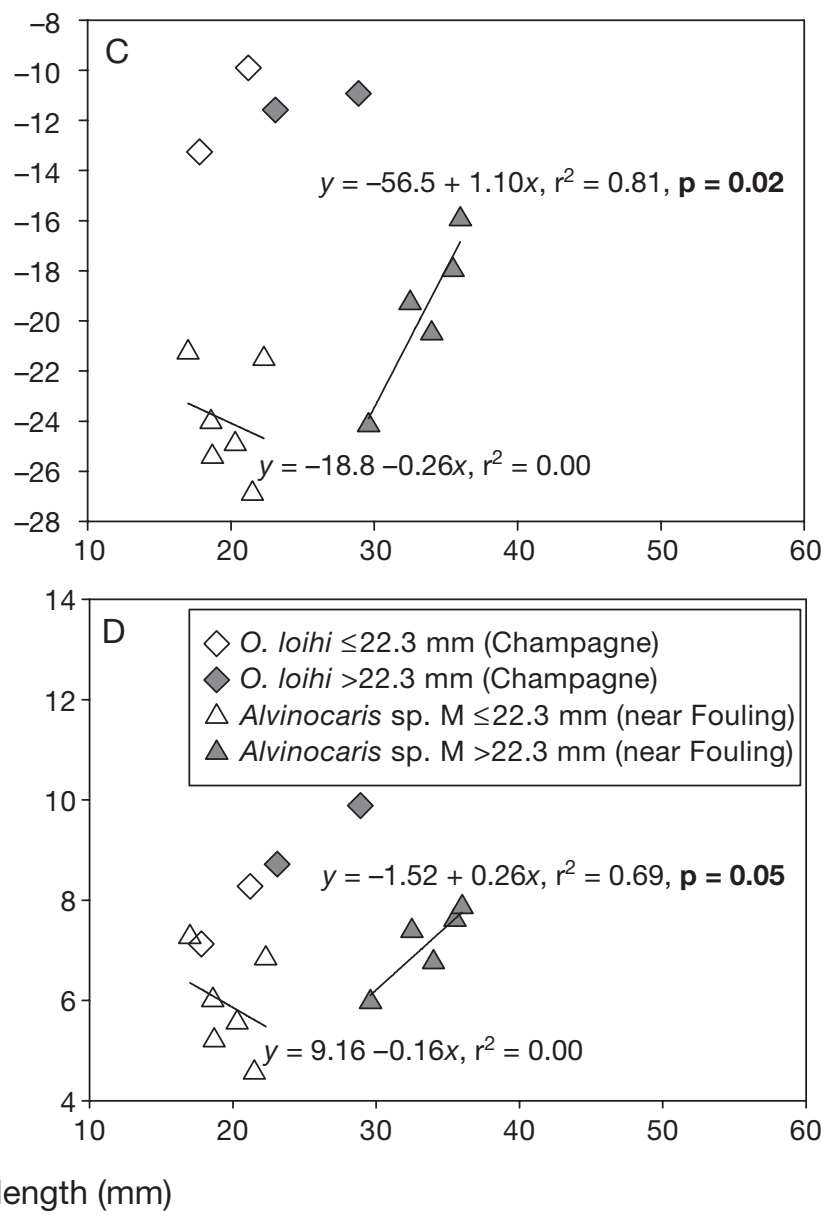

Fig. 3. Opaepele loihi and Alvinocaris sp. M. Relationships between $\delta^{13} \mathrm{C}, \delta^{15} \mathrm{~N}$ and juvenile total length (telson to tip of rostrum; $\mathrm{mm})$ at NW Rota-1 $(\mathrm{A}, \mathrm{B})$ and NW Eifuku $(\mathrm{C}, \mathrm{D})$. $\mathrm{r}^{2}$ values are 'adjusted' $\mathrm{r}^{2}$. No adults of either species - which can exceed $5 \mathrm{~cm}$ from eye to telson - were analyzed in this study. The exact transition size between juveniles and adults is unknown, but it is $>4 \mathrm{~cm}$ (eye to telson; V. Tunnicliffe pers. comm.)

tionship between $\delta^{13} \mathrm{C}$ level and total length for $O$. loihi $\leq 25 \mathrm{~mm}$ (adjusted $\mathrm{r}^{2}=0.27, \mathrm{p}=0.01, \mathrm{n}=19$ ), but no analogous relationship for shrimp $>25 \mathrm{~mm}$ (adjusted $\left.\mathrm{r}^{2}=0.04\right)$, where mean $\delta^{13} \mathrm{C}$ was $-12.1 \% \pm 1.4 \%$ o SD (n =14) (Fig. 3A). There was also a significant positive relationship between size and $\delta^{15} \mathrm{~N}$ values for $O$. loihi $\leq 25 \mathrm{~mm}$ (adjusted $\mathrm{r}^{2}=0.57, \mathrm{p}<0.01, \mathrm{n}=19$ ), but no such relationship was found for individuals $>25 \mathrm{~mm}$ in length (adjusted $\mathrm{r}^{2}=0.00$ ) (Fig. 3B). Mean $\delta^{15} \mathrm{~N}$ for O. loihi $>25 \mathrm{~mm}$ was $10.5 \% \pm 0.3 \%$ o $(\mathrm{n}=14)$. Bacterial mats, as well as detritus from Shimmering Vent, were considerably more depleted in ${ }^{13} \mathrm{C}$ than the majority of the O. loihi samples (Fig. 4). One small O. loihi individual $(16.3 \mathrm{~mm})$ had a carbon isotopic signature that matched those of the 2 particulate samples and that of a detritus sample from Gastros. However, almost all O. loihi were more enriched in ${ }^{13} \mathrm{C}$ than any of the potential food sources sampled.
NW Eifuku: Lipids. Juvenile Opaepele loihi ranged in total length from 17.8 to $28.9 \mathrm{~mm}$ at the Champagne site on NW Eifuku while near Fouling, Alvinocaris sp. $\mathrm{M}$ were between 17.0 and $36.0 \mathrm{~mm}$ (Table 1). O. loihi and Alvinocaris sp. M both showed a strong ontogenetic loss in relative amounts of PUFA in neutral lipid (Fig. 2E; Tables 4 \& 5). Again there was a threshold size above which PUFA content fell sharply, in this case $\sim 22 \mathrm{~mm}$. As at NW Rota-1, the most abundant PUFA was $22: 6 \omega 3$, ranging from 4.1 to $21.0 \%$ of the neutral fatty acids in shrimp of both species $\leq 22.3 \mathrm{~mm}$.

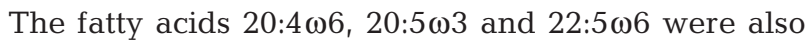
relatively abundant in the small shrimp. As shrimp length increased, so did relative amounts of $\omega 7$ MUFA, $\omega 4$ dienes and OBFA (Fig. 2F-H). In Alvinocaris sp. M, however, relative amounts of $\omega 7$ MUFA appeared to level off above $30 \mathrm{~mm}$ (Fig. 2F) and for a given size, $\omega 4$ diene levels were always lower compared to O. loihi 


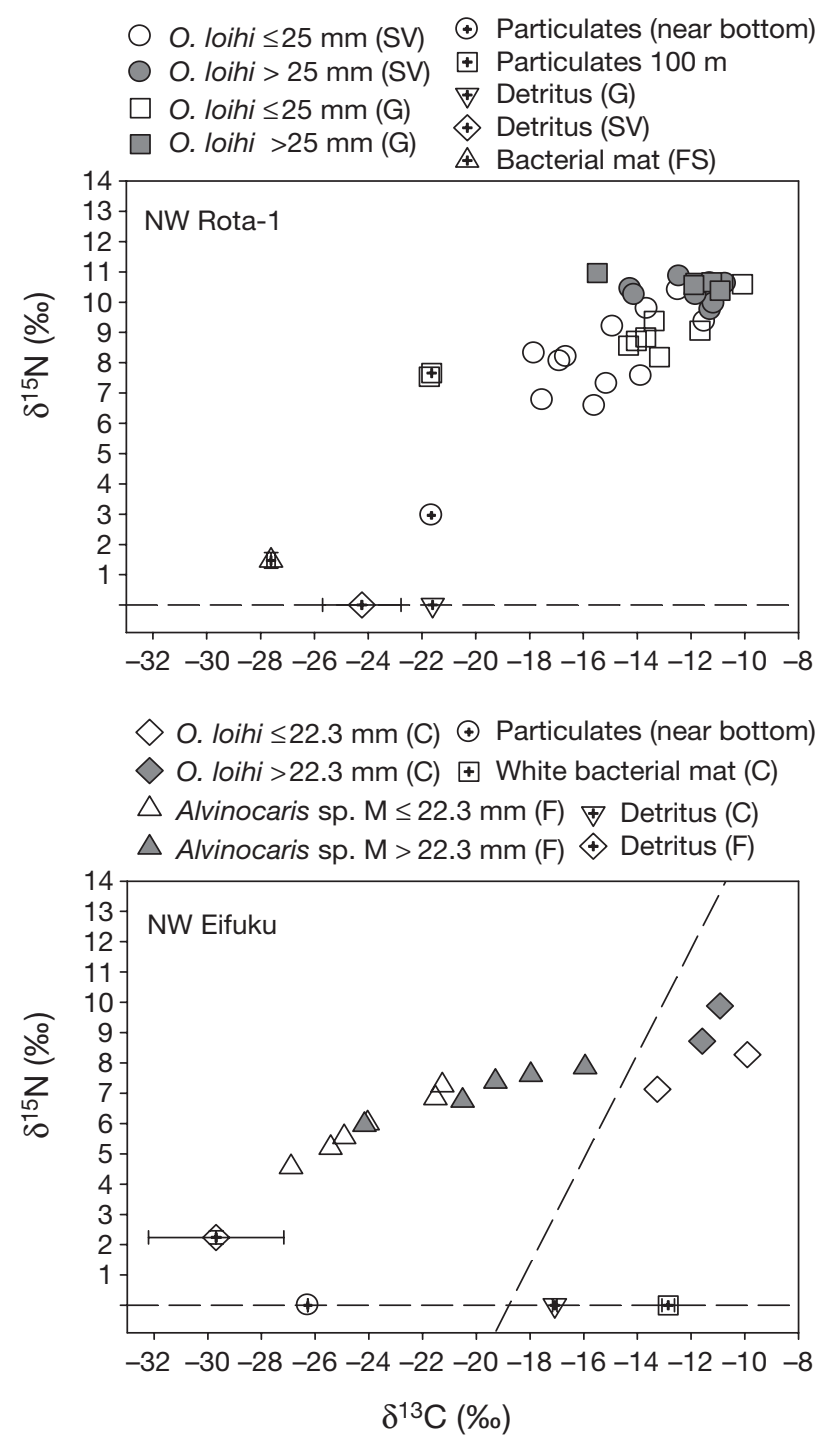

Fig. 4. Opaepele loihi and Alvinocaris sp. M. $\delta^{13} \mathrm{C}$ and $\delta^{15} \mathrm{~N}$ levels in juveniles at NW Rota-1 and NW Eifuku. End members along the horizontal dashed line were present in quantities too small to enable measurement of $\delta^{15} \mathrm{~N}$. The diagonal line in the NW Eifuku graph indicates that the 2 sites are geographically and chemically distinct. SV: Shimmering Vent; G: near Gastros; FS: Fault Shrimp; C: Champagne; F: near Fouling

from the Champagne site (Fig. 2G). The largest Alvinocaris sp. M specimen (36.0 mm) had a PUFA-rich polar lipid fraction, compared to the neutral lipid that was depleted in PUFA (Table 5, last 2 columns). Polar

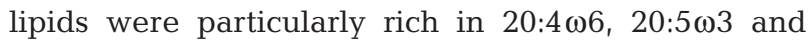
22:6 $\omega 3$. The difference in relative amounts of PUFA between neutral and polar lipid fractions was more obvious in Alvinocaris sp. M than in O. loihi from NW Rota-1. Also, the polar lipid in this specimen was quite poor in 18:1 107 and was characterized by the lowest relative amount of this fatty acid in any sample in this study $(2.8 \%$ of the polar fatty acids).
Table 4. Opaepele loihi at NW Eifuku, Champagne site. Percentage (\%) fatty acid composition of neutral lipid in juveniles of increasing length (SFA: saturated fatty acids; MUFA: monounsaturated fatty acids; PUFA: polyunsaturated fatty acids)

\begin{tabular}{|c|c|c|c|c|}
\hline \multirow[t]{2}{*}{ Fatty acid } & \multicolumn{4}{|c|}{ - Length $(\mathrm{mm})$} \\
\hline & 17.8 & 21.2 & 23.1 & 28.9 \\
\hline $14: 1$ & 2.2 & 3.4 & 5.3 & 5.3 \\
\hline $14: 0$ & 1.2 & 2.5 & 3.1 & 2.8 \\
\hline $\mathrm{i}-15: 0$ & 0.1 & 0.1 & 0.3 & 0.2 \\
\hline ai-15:0 & 0.1 & 0.1 & 0.2 & 0.2 \\
\hline $15: 0$ & & & & 0.0 \\
\hline $\mathrm{i}-16: 0$ & & 0.0 & 0.1 & 0.1 \\
\hline $16: 1 \omega 7$ & 18.1 & 26.9 & 35.9 & 36.7 \\
\hline $16: 2 \omega 4$ & 0.7 & 1.5 & 2.0 & 1.8 \\
\hline $16: 0$ & 3.6 & 8.0 & 10.9 & 13.9 \\
\hline $\mathrm{i}-17: 0$ & 0.2 & 0.3 & 0.4 & 0.3 \\
\hline $17: 0$ & 0.2 & 0.1 & 0.2 & 0.1 \\
\hline $18: 2 \omega 6$ & 1.5 & 1.0 & 1.9 & 1.7 \\
\hline $18: 1 \omega 9+\omega 13$ & 11.6 & 5.1 & 6.7 & 10.9 \\
\hline $18: 1 \omega 7$ & 6.5 & 13.6 & 14.3 & 12.1 \\
\hline $18: 2 \omega 4$ & 1.7 & 4.3 & 6.8 & 5.1 \\
\hline $18: 0$ & 0.8 & 1.2 & 2.3 & 1.7 \\
\hline $20: 4 \omega 6$ & 8.7 & 4.2 & 1.1 & 1.1 \\
\hline $20: 5 \omega 3$ & 5.6 & 3.5 & 1.2 & 0.5 \\
\hline $20: 2 \Delta 5,11$ & & & & 0.0 \\
\hline $20: 2 \Delta 5,13$ & 0.6 & 0.8 & 0.3 & 0.2 \\
\hline $20: 2 \Delta 13,16$ & 0.5 & 0.2 & 0.4 & 0.4 \\
\hline $20: 1 \omega 13+\omega 9$ & 0.2 & 0.2 & 0.4 & 0.2 \\
\hline $20: 1 \omega 7$ & 0.2 & 0.5 & 0.4 & 0.4 \\
\hline $22: 5 \omega 6$ & 9.8 & 8.3 & 2.1 & 1.2 \\
\hline $22: 6 \omega 3$ & 20.7 & 12.2 & 2.2 & 1.8 \\
\hline $22: 4$ & 0.7 & 0.3 & 0.2 & 0.1 \\
\hline $22: 5 \omega 3$ & 2.1 & 0.8 & 0.2 & 0.2 \\
\hline $22: 2 \Delta 7,15$ & 0.9 & & & 0.1 \\
\hline $22: 1 \omega 11$ & 0.3 & & & 0.1 \\
\hline $22: 1 \omega 9$ & 0.4 & & & 0.1 \\
\hline $22: 1 \omega 7$ & 0.7 & 0.9 & 1.0 & 0.7 \\
\hline SFA & 6.2 & 12.3 & 17.5 & 19.3 \\
\hline MUFA & 40.2 & 50.5 & 64.0 & 66.5 \\
\hline Dienes & 5.9 & 7.8 & 11.4 & 9.2 \\
\hline PUFA & 47.6 & 29.4 & 7.1 & 4.9 \\
\hline
\end{tabular}

NW Eifuku: Stable isotopes. Both juvenile Alvinocaris sp. M and Opaepele loihi generally became more enriched in ${ }^{13} \mathrm{C}$ and ${ }^{15} \mathrm{~N}$ with increasing size, the latter species almost always more enriched in the 2 isotopes than the former (Fig. 3C, D). Small Alvinocaris sp. M $(\leq 22.3 \mathrm{~mm})$ were quite depleted in ${ }^{13} \mathrm{C}$, with $\delta^{13} \mathrm{C}$ levels ranging between -26.9 and $-21.3 \%$; $\delta^{15} \mathrm{~N}$ ranged from 4.6 to $7.3 \%$. In the larger Alvinocaris sp. M $(>22.3 \mathrm{~mm}), \delta^{13} \mathrm{C}$ levels ranged between -24.2 and $-16.0 \%$ and $\delta^{15} \mathrm{~N}$ from 6.0 to $7.9 \%$. There was a strong positive relationship between total length and $\delta^{13} \mathrm{C}$ levels in Alvinocaris sp. $\mathrm{M}>22.3 \mathrm{~mm}$ (adjusted $\mathrm{r}^{2}=$ 0.81, $\mathrm{p}=0.02, \mathrm{n}=5$ ), and between size and $\delta^{15} \mathrm{~N}$ (adjusted $\mathrm{r}^{2}=0.69, \mathrm{p}=0.05, \mathrm{n}=5$ ), although the latter was not significant at $\alpha<0.05$. For Alvinocaris sp. M $\leq 22.3 \mathrm{~mm}$, isotopic composition was not related to size (adjusted $\mathrm{r}^{2}=0.00$ ); mean $\delta^{13} \mathrm{C}$ was $-24.0 \%$ o $\pm 2.2 \%$ o 
Table 5. Alvinocaris sp. M at NW Eifuku, near Fouling. Percentage (\%) fatty acid composition of neutral lipid in juveniles of increasing length, and of polar lipid (values italicized) in the smallest (17.0 mm) and largest (36.0 mm) individuals (SFA: saturated fatty acids; MUFA: monounsaturated fatty acids; PUFA: polyunsaturated fatty acids)



$(\mathrm{n}=6)$ and $\delta^{15} \mathrm{~N}, 5.9 \% \pm 1.0 \%$ o $(\mathrm{n}=6)$. It was not possible to perform regression analyses between isotopic composition and size for PUFA-rich and PUFA-poor ( $\leq$ or $>22.3 \mathrm{~mm}$ ) O. loihi from NW Eifuku, as there were too few samples. In general, the isotopic composition of O. loihi from NW Eifuku was very similar to that at NW Rota-1. Near Fouling, some of the Alvinocaris sp. M $\leq 22.3 \mathrm{~mm}$ had $\delta^{13} \mathrm{C}$ signatures within the range of levels measured in detritus, and had similar carbon isotopic values to particulates collected near the sea floor (Fig. 4). Two of the other small specimens (17.0 and $22.3 \mathrm{~mm}$ ) had $\delta^{13} \mathrm{C}$ signatures of $\sim-22 \%$. Alvinocaris sp. $M>22.3 \mathrm{~mm}$ were more enriched in ${ }^{13} \mathrm{C}$ than any of the potential foods. At Champagne, detritus was more depleted in ${ }^{13} \mathrm{C}$ than $O$. loihi, while a white bacterial mat had a similar $\delta^{13} \mathrm{C}$ signature.

\section{DISCUSSION}

\section{Diets of alvinocaridid larvae - extrapolation from small juveniles}

Small Opaepele loihi and Alvinocaris sp. M juveniles were rich in PUFA, as has been reported for postlarvae and juveniles of MAR alvinocaridids (Pond et al. 1997a, 2000, Allen et al. 1998, 2001, Dixon et al. 1998). Published isotopic data on alvinocaridid juveniles corroborate the lipid data, showing that migratory postlarvae feed on material derived from pelagic photosynthesis (Polz et al. 1998, Gebruk et al. 2000, Vereshchaka et al. 2000, Van Dover 2002). Moreover, some of the smallest shrimp of both species in this study had isotopic values in the range of those 
expected given a photosynthetic origin (i.e. $\delta^{13} \mathrm{C}$ : $\sim-22 \%$, $\delta^{15} \mathrm{~N}: \sim 7 \%$, Gebruk et al. 2000). The dispersal phase of alvinocaridids has been widely observed, as postlarvae have been found at midwater depths above most vent sites at which adults are present (discussed by Herring 2006). Shrimp may adopt this dispersal strategy to obtain the PUFA essential for growth and development (Sargent et al. 1995), and to promote genetic diversity and colonize new vents (Tyler \& Young 2003).

We propose that the observed decrease in proportions of PUFA in Opaepele loihi and Alvinocaris sp. M represents gradual biochemical changes associated with a switch from planktonic feeding by postlarvae to feeding by settled juveniles at the vent sites. This is especially clear in $O$. loihi from NW Rota-1, where shrimp $\leq 25 \mathrm{~mm}$ showed significant ${ }^{13} \mathrm{C}$ and ${ }^{15} \mathrm{~N}$ enrichment with increases in total length, whereas there was no analogous relationship for those $>25 \mathrm{~mm}$. Relationships between $\delta^{13} \mathrm{C}$ and $\delta^{15} \mathrm{~N}$ and size were less clear in small Alvinocaris sp. M juveniles from NW Eifuku $(\leq 22.3 \mathrm{~mm})$, perhaps due to a more varied diet (discussed below). The ontogenetic patterns observed in our work may represent the switch from food depleted in ${ }^{13} \mathrm{C}$ (phytoplankton) to the more ${ }^{13} \mathrm{C}$ enriched chemoautotrophic bacteria thought to form the base of the food web at many vent sites (sensu Van Dover 2002).

All alvinocaridid juveniles analysed so far, including Opaepele loihi and Alvinocaris sp. M, contain significant amounts of 22:6 $\omega 3$ and 20:5 $\omega 3$ (Pond et al. 1997a, 2000, Allen et al. 1998, 2001). Such a lipid composition suggests a larval diet rich in phytoplankton, including diatoms and flagellates (Dalsgaard et al. 2003). However, the water column in the vicinity of the Mariana Arc is frequently oligotrophic and dominated by picoplankton $(<2 \mu \mathrm{m})$ such as heterotrophic bacteria, cyanobacteria, procholorophytes and picoeukaryotes (Yamaguchi et al. 2002). With the exception of some prymnesiophytes and ciliates $>2 \mu \mathrm{m}$ (Pond \& Harris 1996), fatty acids in these planktonic groups generally contain small amounts of the main PUFA found in our small shrimp juveniles (Viso \& Marty 1993). Furthermore, particulate matter over the summit of NW Rota-1 (100 $\mathrm{m}$ ) was dominated by 16:0 and 18:0 fatty acids (27 and $55 \%$ of the total, respectively), with only small

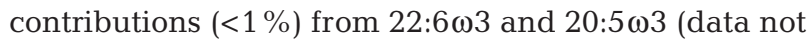
shown), which indicates oligotrophy. Although we can say little with such limited planktonic sampling around the Mariana Arc, this region of the Pacific is generally characterized by weak seasonal cycles and low abundances of phytoplankton and mesozooplankton (Limsakul et al. 2002).

How then do we account for the apparent mismatch between PUFA accumulation in larval shrimp and the low abundances of these compounds in the pelagic zone for much of the year? One explanation is that alvinocaridids are capable of intense PUFA sequestration as larvae. They likely feed on the available plankton, either in the photic zone or at hydrothermal plume depth, where descending material, lipid-rich ascending particles, and zooplankton accumulate (Cowen et al. 2001), and amass the PUFA essential for structural and reproductive purposes. Embryos of non-vent shrimp preferentially conserve PUFA and use MUFA to fuel early growth and development (Morais et al. 2002). Such conservation of PUFA presumably buffers shrimp against the paucity of PUFA at vent sites. It has also been suggested that bacteria at hydrothermal vents are capable of synthesizing PUFA and could represent a local source (Pond et al. 2002). Some bacteria can produce PUFA in large quantities (Nichols \& McMeekin 2002), but there is no evidence that these strains grow at vents. Moreover, stable isotope measurements of individual PUFA in alvinocaridid juveniles reflect a photic zone origin (Pond et al. 1997a, 2000). We therefore assert that the PUFA found in the small juvenile shrimp in this study is dietary and originated during a migratory pelagic larval phase.

\section{Diets of settled alvinocaridid juveniles}

At the vent sites on NW Rota-1, Opaepele loihi juveniles are probably eating bacteria. This is apparent in the neutral lipid data, as proportions of $\Sigma \omega 7$ MUFA, $\Sigma \omega 4$ dienes, and $\Sigma$ OBFA increased with size, and PUFA proportions decreased as the photosynthetic signal was gradually lost. Microbial mats from gas hydrates are composed almost entirely of 16:1 17 and 18:1 1 7 (Zhang et al. 2005). OBFA have been used to indicate a broad bacterial diet in hydrothermal vent gastropods (Pranal et al. 1996); increases in these fatty acids in shrimp tissues may signify the ingestion of diverse microbial products at vent sites. The fatty

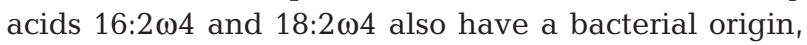
as both may be synthesized by bacteria via desaturation and chain elongation of 16:107 (Pond et al. 1997b). However, bacterial feeding indices, in particular $\Sigma \omega 4$ dienes, are lower in $O$. loihi than in MAR shrimp that graze primarily on their episymbionts (Pond et al. 1997b). This difference suggests that $O$. loihi may be less dependent on bacteria than symbiotic MAR species; on occasion $O$. loihi adults were observed scavenging dead fish (Tunnicliffe et al. unpubl. data).

The isotope data also suggest a primarily bacterivorous feeding mode for juvenile Opaepele loihi at NW Rota-1, with $\delta^{13} \mathrm{C}$ values levelling off at about $-12 \%$ in 
the larger individuals (>25 mm). As in MAR shrimp with episymbionts (Gebruk et al. 2000), the isotopic composition of $O$. loihi is consistent with a diet based on bacteria containing form II RuBisCo, which fractionates $\mathrm{CO}_{2}$ to a lesser degree ('-11\%o group') than form I RuBisCo ('-30\% group') (Robinson \& Cavanaugh 1995). However, the bacterial mats abundant at vents generally have depleted carbon signatures, more consistent with form I RuBisCo (Gebruk et al. 2000). A mat sample from NW Rota-1 is too depleted in both isotopes $\left(\delta^{13} \mathrm{C}:-27.6 \%\right.$, $\delta^{15} \mathrm{~N}: 1.5 \%$ ) to represent a primary food source to $O$. loihi. It is unlikely that $O$. loihi eats detritus, as this potential food item is also too depleted in ${ }^{13} \mathrm{C}$. Similar observations in this and other vent systems have led researchers to invoke a missing end member, one with $\delta^{13} \mathrm{C}$ values of -10 to $-15 \%$ and a $\delta^{15} \mathrm{~N}$ of -1 to $5 \%$ (Van Dover 2002, Limén et al. 2006). Limén et al. (2006) have suggested that high turnover rates of ${ }^{13} \mathrm{C}$-enriched organic material greatly limits in situ accumulation and effectively precludes the sampling of the chemosynthetic end member responsible for the enriched isotopic compositions of many vent fauna.

Opaepele loihi at NW Eifuku had a neutral lipid composition very similar to $O$. loihi on NW Rota-1; these 2 populations are probably feeding on similar types of bacteria. Detritus from the Champagne site was too depleted in ${ }^{13} \mathrm{C}$ and ${ }^{15} \mathrm{~N}$ to be an important food source to $O$. loihi, but a white bacterial mat had a very similar $\delta^{13} \mathrm{C}$ value of $\sim 13 \%$. This sample could represent a bacterial mat with form II RuBisCo that may also feature prominently in the food web on NW Rota-1. It is unfortunate that we cannot take this argument further, as the mat sample was too small to permit a $\delta^{15} \mathrm{~N}$ measurement. Furthermore, the vent fluid at Champagne was unusual in that it was supersaturated with $\mathrm{CO}_{2}$ (Limén \& Juniper 2006). Such conditions may affect the fractionation of $\mathrm{CO}_{2}$, making it difficult to identify the dominant form of RuBisCo present (discussed in Limén \& Juniper 2006).

There were a few key differences in the lipid profiles of Alvinocaris sp. M and Opaepele loihi at NW Eifuku. Relative amounts of $\omega 7$ MUFA seem to decrease in larger Alvinocaris sp. M individuals, and $\omega 4$ levels were always lower in this species relative to O. loihi. The polar lipid fraction of the largest Alvinocaris sp. M sampled (36.0 mm) was particularly poor in 18:1 $1 \omega 7$ and rich in 18:1 $\omega 9$, as compared to $O$. loihi polar lipids. These observations suggest a lesser dependence on bacteria for Alvinocaris sp. M and the in situ distribution of the 2 species corroborates this idea. O. loihi is usually found actively foraging on areas of vigorous hydrothermal discharge and substantial bacterial mat growth, where it grazes on bacterial filaments, while Alvinocaris sp. M prefers rock outcrops or mussel beds with diffuse flow (Tunnicliffe et al. unpubl. data). Unfortunately, adults were very difficult to sample. We would expect to see stronger differences in fatty acid composition with age, since field observations indicate that Alvinocaris sp. M becomes more carnivorous and aggressive, whilst $O$. loihi probably continues primarily as a grazer of bacterial filaments (Tunnicliffe et al. unpubl. data). Adult Alvinocaris sp. M on NW Rota-1 were also observed feeding on dead pelagic shrimp and midwater fish killed by toxic hydrothermal plumes (Tunnicliffe et al. unpubl. data). These authors suggest that such 'fish kills' may figure prominently in the hydrothermal vent food webs associated with active volcanic arcs.

Small Alvinocaris sp. M juveniles at NW Eifuku were generally the most isotopically depleted shrimp sampled in this study. A couple had clear planktonic signals (lipids and isotopes), but the ones more depleted in ${ }^{13} \mathrm{C}$ and ${ }^{15} \mathrm{~N}$ had diets that could have included detritus, near-bottom particulate matter, and a form I RuBisCo bacterial mat, such as that sampled on NW Rota-1. Tunnicliffe et al. (unpubl. data) have suggested that the more peripheral habitats frequented by Alvinocaris sp. M favour bacteria with form I RuBisCo over those with form II. Although we did not sample it, mussel-derived material (pseudofaeces and mucous) could also feature in the diet of Alvinocaris sp. $\mathrm{M}$ at NW Eifuku; MAR alvinocaridids are thought to ingest such material (Pond et al. 1997a). Bathymodiolid mussels are among the most depleted organisms in vent communities, with $\delta^{13} \mathrm{C}$ values ranging between $-25 \%$ and $-35 \%$ (Van Dover 2002). The positive relationships between $\delta^{13} \mathrm{C}, \delta^{15} \mathrm{~N}$ and size in Alvinocaris sp. $\mathrm{M}>22.3 \mathrm{~mm}$ could represent a switch to a more isotopically enriched food item, but probably not the form II RuBisCo chemoautotrophic bacteria implied for Opaepele loihi. Instead, this alternate food source could be other invertebrates and fish carcasses. This is in keeping with the opportunistic, scavenging feeding mode employed by A. markensis on the MAR (Pond et al. 1997b, Gebruk et al. 2000).

Acknowledgements. We thank chief scientist Dr. B. Embley and other members of the 2004 Pacific Ring of Fire expedition for their collaboration in the field. We also thank the officers and crew of the RV 'Thomas G. Thompson', and the 'ROPOS' pilots for help with the sample collection. Dr. J. McKay and A. Adamowicz performed the stable isotope analyses. M.H. Veilleux helped with sample analysis and A. Ouellet developed the GC column program. Dr. J. Dower provided the particulate sample taken at $100 \mathrm{~m}$ above NW Rota-1. Drs. S. Budge and C. Parrish provided valuable methodological advice. S. Merle (NOAA Vents Program) provided the map in Fig. 1. NSERC Canada grants to Drs. V. Tunnicliffe and S.K.J., and an NSERC PDF fellowship and Royal Society of London Travel Award to C.J.S. supported this work. 


\section{LITERATURE CITED}

Allen CE, Tyler PA, Varney MS (1998) Lipid profiles of hydrothermal vent shrimps. Cah Biol Mar 39:229-231

Allen CE, Copley JT, Tyler PA (2001) Lipid partitioning in the hydrothermal vent shrimp Rimicaris exoculata. Mar Ecol 22:241-253

Budge SM, Parrish CC (2003) FA determination in cold water marine samples. Lipids 38:781-791

Casanova B, Brunet M, Segonzac M (1993) L'impact d'une épibiose bactérienne sur la morphologie fonctionnelle de crevettes associées à l'hydrothermalisme médioAtlantique. Cah Biol Mar 34:573-588

Christie WW (2003) Lipid analysis. Isolation, separation, identification and structural analysis of lipids, 3rd edn. PJ Barnes \& Associates: The Oily Press, Bridgewater

Cowen JP, Bertram MA, Wakeham SG, Thomson RE, Lavelle JW, Baker ET, Feely RA (2001) Ascending and descending particle flux from hydrothermal plumes at Endeavor Segment, Juan de Fuca Ridge. Deep-Sea Res I 48:1093-1120

Dalsgaard J, St. John M, Kattner G, Müller-Navarra D, Hagen W (2003) Fatty acid trophic markers in the pelagic marine environment. Adv Mar Biol 46:225-340

Dixon DR, Dixon LRJ, Pond DW (1998) Recent advances in our understanding of the life history of bresiliid vent shrimps on the MAR. Cah Biol Mar 39:383-386

Embley RW, Chadwick WW, Baker ET, Butterfield DA and others (2006) Long-term eruptive activity at a submarine arc volcano. Nature 441:494-497

Fullarton JG, Dando PR, Sargent JR, Southward AJ, Southward EC (1995) Fatty acids of hydrothermal vent Ridgeia piscesae and inshore bivalves containing symbiotic bacteria. J Mar Biol Assoc UK 75:455-468

Gebruk AV, Southward EC, Kennedy H, Southward AJ (2000) Food sources, behaviour, and distribution of hydrothermal vent shrimps at the Mid-Atlantic Ridge. J Mar Biol Assoc UK 80:485-499

Herring PJ (2006) Presence of postlarval alvinocaridid shrimps over southwest Indian Ocean hydrothermal vents, with comparisons of the pelagic biomass at different vent sites. J Mar Biol Assoc UK 86:125-128

Kharlamenko VI, Zhukova NV, Khotimchenko SV, Svetashev VIKGM (1995) Fatty acids as markers of food sources in a shallow-water hydrothermal ecosystem (Kraternaya Bight, Yanich Island, Kurile Islands). Mar Ecol Prog Ser 120:231-241

Lee RF, Nevenzel JC, Paffenhöfer GA (1971) Importance of wax esters and other lipids in the marine food chain: phytoplankton and copepods. Mar Biol 9:99-108

Limén H, Juniper SK (2006) Habitat controls on vent food webs at Eifuku Volcano, Mariana Arc. Cah Biol Mar 47: $449-455$

Limén H, Juniper SK, Tunnicliffe V, Clément M (2006) Benthic community structure on two peaks of an erupting seamount: Northwest Rota-1 Volcano, Mariana Arc, western Pacific. Cah Biol Mar 47:457-463

Limén H, Stevens CJ, Bourass Z, Juniper SK. (in press) The trophic ecology of siphonostomatoid copepods at deep-sea hydrothermal vents in the North Pacific. Mar Ecol Prog Ser

Limsakul A, Saino T, Goes JI, Midorikawa T (2002) Seasonal variability in the lower trophic level environments of the western subtropical Pacific and Oyashio Waters - a retrospective study. Deep-Sea Res II 49:5487-5512

Martin JW, Haney TA (2005) Decapod crustaceans from hydrothermal vents and cold seeps: a review through 2005. Zool J Linn Soc Lond 145:445-522
Morais S, Narcisco L, Calado R, Nunes ML, Rosa R (2002) Lipid dynamics during the embryonic development of Plesionika martia martia (Decapoda; Pandalidae), Palaemon serratus and P. elegans (Decapoda; Palaemonidae): relation to metabolic consumption. Mar Ecol Prog Ser 242: 195-204

Nichols DS, McMeekin TA (2002) Biomarker techniques to screen for bacteria that produce polyunsaturated fatty acids. J Microbiol Meth 48:161-170

Nyssen F, Brey T, Dauby P, Graeve M (2005) Trophic position of Antarctic amphipods-enhanced analysis by a 2-dimensional biomarker assay. Mar Ecol Prog Ser 300: $135-145$

Parrish CC (1999) Determination of total lipid, lipid classes, and fatty acids in aquatic samples. In: Arts MT, Wainman BC (eds) Lipids in freshwater ecosystems. SpringerVerlag, New York, p 4-20

Polz MF, Robinson JJ, Cavanaugh CM, Van Dover CL (1998) Trophic ecology of massive shrimp aggregations at a MidAtlantic Ridge hydrothermal vent site. Limnol Oceanogr 43:1631-1638

Pond DW, Harris RP (1996) The lipid composition of the coccolithophore Emiliania huxleyi and its possible ecophysiological significance. J Mar Biol Assoc UK 76:579-594

Pond DW, Segonzac M, Bell MV, Dixon DR, Fallick AE, Sargent JR (1997a) Lipid and lipid carbon stable isotope composition of the hydrothermal vent shrimp Mirocaris fortunata: evidence for nutritional dependence on photosynthetically fixed carbon. Mar Ecol Prog Ser 157:221-231

Pond DW, Dixon DR, Bell MV, Fallick AE, Sargent JR (1997b) Occurrence of 16:2(n-4) and 18:2(n-4) fatty acids in the lipids of the hydrothermal vent shrimps Rimicaris exoculata and Alvinocaris markensis: nutritional and trophic implications. Mar Ecol Prog Ser 156:167-174

Pond DW, Bell MV, Dixon DR, Fallick AE, Segonzac M, Sargent JR (1998) Stable-carbon-isotope composition of fatty acids in hydrothermal vent mussels containing methanotrophic and thiotrophic bacterial endosymbionts. Appl Environ Microbiol 64:370-375

Pond DW, Gebruk A, Southward EC, Southward AJ, Fallick AE, Bell MV, Sargent JR (2000) Unusual fatty acid composition of storage lipids in the bresilioid shrimp Rimicaris exoculata couples the photic zone with MAR hydrothermal vent sites. Mar Ecol Prog Ser 198:171-179

Pond DW, Allen CE, Bell MV, Van Dover CL, Fallick AE, Dixon DR, Sargent JR (2002) Origins of long-chain polyunsaturated fatty acids in the hydrothermal vent worms Ridgeia piscesae and Protis hydrothermica. Mar Ecol Prog Ser 225:219-226

Post DM (2002) Using stable isotopes to estimate trophic position: models, methods and assumptions. Ecology 83: 703-718

Pranal V, Fiala-Médioni A, Guezennec J (1996) Fatty acid characteristics in two symbiotic gastropods from a deep hydrothermal vent of the West Pacific. Mar Ecol Prog Ser 142:175-184

Robinson JJ, Cavanaugh C (1995) Expression of form I and form II Rubisco in chemoautotrophic symbioses: implications for the interpretation of stable carbon isotope values. Limnol Oceanogr 40:1496-1502

Sargent JR, Bell MV, Bell JG, Henderson RJ (1995) Origins and functions of $n-3$ polyunsaturated fatty acids in marine organisms. In: Cleve G, Paltauf F (eds) Phospholipid characterization, metabolism and novel biological applications. American Oil Society Press, Champaign, IL, p 248-259

Segonzac M, de Saint Laurent M, Casanova B (1993) L'énigme du comportement trophique des crevettes 
Alvinocarididae des sites hydrothermaux de la dorsale médio-atlantique. Cah Biol Mar 34:535-571

Stübing D, Hagen W (2003) Fatty acid biomarker ratios-suitable trophic indicators in Antarctic euphausiids? Polar Biol 26:774-782

Suhr SB, Pond DW, Gooday AJ, Smith CR (2003) Selective feeding by benthic foraminifera on phytodetritus on the western Antarctic Peninsula shelf: evidence from fatty acid biomarker analysis. Mar Ecol Prog Ser 262: 153-162

Tyler PA, Young CM (2003) Dispersal at hydrothermal vents: a summary of recent progress. Hydrobiologia 503:9-19

Van Dover CL (2002) Trophic relationships among invertebrates at the Kairei hydrothermal vent field (Central Indian Ridge). Mar Biol 141:761-772

Van Dover CL, Fry B (1989) Stable isotopic compositions of hydrothermal vent organisms. Mar Biol 102:257-263

Van Dover CL, Fry B, Grassle JF, Humphris S, Rona PA (1988)

Editorial responsibility: Otto Kinne,

Oldendorf/Luhe, Germany
Feeding biology of the shrimp Rimicaris exoculata at hydrothermal vents on the Mid-Atlantic Ridge. Mar Biol 98:209-216

Vereshchaka AL, Vinogradov GM, Lein A Yu, Dalton $\mathrm{S}$, Dehairs F (2000) Carbon and nitrogen isotopic composition of the fauna from the Broken Spur hydrothermal vent field. Mar Biol 136:11-17

Viso AC, Marty JC (1993) Fatty acids from 28 marine microalgae. Phytochemistry 34:1521-1533

Yamaguchi A, Watanabe $\mathrm{Y}$, Ishida $\mathrm{H}$, Harimoto $\mathrm{T}$ and others (2002) Structure and size distribution of plankton communities down to the greater depths in the western North Pacific Ocean. Deep-Sea Res II 49:5513-5529

Zhang CL, Huang Z, Cantu J, Pancost RD, Brigmon RL, Lyons TW, Sassen R (2005) Lipid biomarkers and carbon isotope signatures of a microbial (Beggiatoa) mat associated with gas hydrates in the Gulf of Mexico. Appl Environ Microbiol 71:2106-2112

Submitted: June 8, 2007; Accepted: September 28, 2007 Proofs received from author(s): February 13, 2008 